\title{
Study on Intestinal Symbiotic Microorganisms and Its Immunity
}

\author{
Qingfeng Wan, Tingyu Wu, Man Ma \\ Jiangxi Medical College, Shangrao, Jiangxi, 334000
}

Keywords: immunity system; intestinal symbiotic microorganisms

\begin{abstract}
There are hundreds of millions of microorganisms in the intestinal tract of animals. These symbiotic microorganisms can help the animals digest and metabolize and maintain intestinal homeostasis. However, microorganisms and their metabolites can also act as antigens to affect the normal function of the intestine. Under normal circumstances, the intestinal immune system can accurately identify symbiotic microorganisms and their metabolites, make them immune tolerant, and maintain homeostasis; in addition, the immune system of the intestine can also avoid the reaction to harmless antigens, and the waste of immune resources. Immune tolerance has been widely used in clinical medicine to reduce the incidence of rejection after organ transplantation and reduce the immune rejection of the uterus to the fetus. However, there are still few reports on how to use the immune tolerance mechanism to slow the ruminal acidosis in ruminants, increase the efficiency of synthesis and utilization of rumen microbial proteins, and improve the feeding regulations for probiotics. To this end, this paper describes the general concepts and applications of immune tolerance, the composition and function of the intestinal immune system, the immunogenicity of intestinal commensal microorganisms, and the mechanism of intestinal immune tolerance.
\end{abstract}

\section{Introduction}

The normal number of intestinal micro-organisms in humans ranges from 1012 to 1014. Their average mass is about $1.5 \mathrm{~kg}$ [1], and about 6 to 10 groups (3000 species) are composed of microbes. Infants colonize the intestines soon after birth until the gut microbes reach a stable symbiotic group. Intestinal microorganisms are beneficial to the host. In the past 10 years of research, intestinal microbes have been found in human development, intestinal barrier, immune regulation, metabolism, nutrient absorption, toxin excretion, and the occurrence and development of diseases. Play a huge role. Disruption of the intestinal microflora may lead to various diseases such as obesity, cirrhosis, diabetes, cardiovascular diseases, and autism. The main function of the intestinal microflora is to help the host to metabolize, so that the energy and nutrients are better utilized to provide nutrition for the intestinal epithelial cells, enhance the host immune function, and help the host resist pathogens. Recently, a large number of studies have shown that the metabolic function of the intestinal microflora is very important, and the efficiency far exceeds the metabolic function of the liver. For example, intestinal microflora can not only affect the fatty acid composition of the retina and the density of the eye lens, bone, and the formation of intestinal blood vessels; it can also provide necessary nutrients (biotin, vitamin K, butyric acid, etc.) and digestion of dietary fiber. Su. Gut microbes have evolved with spine animals for thousands of years, so the implementation of the immune system's normal function (resisting bacterial pathogens) needs to rely on intestinal microbes. At the same time, intestinal microflora is an important factor that stimulates the maturity of the mucosal immune system (mucosalimmune system) and the systemic immune system. Many experimental studies have found that the composition and metabolites of the intestinal microflora have important effects on immune and inflammatory responses. If the internal immune system of the intestine collapses, chronic enteritis diseases such as Crohn's disease and ulcerative colitis can be caused [2]. However, due to the diversity of commensal intestinal microbes and the fact that it is difficult to determine which bacteria are commensal bacteria Bacteria, so the immune regulation of intestinal microbial colonization is complex. In recent years, the study of intestinal flora and immunity has received more and more attention. 


\section{The Composition and Function of the Intestinal Immune System}

The intestinal immune system is part of the body's immune system and is commonly found in the intestinal mucosa and directly contacts antigens in the external environment. The intestinal immune system not only plays an important role in the identification and clearance of pathogens, but also is an important part of the immune tolerance of foods, symbiotic microorganisms and other beneficial substances [3]. The intestinal immune system is mainly composed of gut-associated lymphoid tissue (GALT). GALT can be divided into two parts from the aspect of morphology and function. The first is GALT composed of mucosal lymph nodes, mainly including Peyer's patches (PP knots) and mesenteric lymph nodes (MLNs). ) is the site of induction of immune responses; followed by GALT consisting of leukocytes widely distributed in the mucosal epithelium and lamina propria, mainly including lamina propria mononuclear cells and intraepithelial lymphocyte (IEL), which is the effect of immune responses. Part [4]. The PP junction is located in the submucosa of the small intestine and contains a large number of B cells, T cells, macrophages (Mø cells) and dendritic cells (DC cells). The PP junction and the intestinal lumen are separated by follicle-associated epitheliium (FAE) cells. Unlike epithelial cells in the mucous membranes, FAE has low levels of digestive enzymes, insignificant brush borders, and no receptors for immunoglobulin (Ig) A. The most prominent feature of FAE is the inclusion of mi-crofold $(\mathrm{M})$ cells. $M$ cells have a high transcellular transport activity, so they are generally considered to be the starting point for enteral immunization systems. MLN is the largest lymph node in the body. Its development is different from that of PP, and it is not influenced by most cytokines, such as tumor necrosis factor (TNF) and receptors. Existing studies suggest that MLN may be a crossroad between the external environment and the mucosal recirculation pathway. The intrinsic layer penetrates a lot of lymphocytes and bone marrow cells. Such as T cells, B cells, Mø cells, neutrophils, other granulocytes and mast cells. These cells have an important role in regulating the immune response. IELs are mainly CD3 $+\mathrm{T}$ cells, followed by secretory immunoglobulin A (SIgA) + B cells and natural killer cells (NK cells). These cells can destroy invading pathogens and denatured cells in vivo through perforin, granzymes, and Fas receptors. In addition, these cells can also regulate other lymphocytes and epithelial cells by secreting interleukin (IL) -22, TNF- $2 \alpha$, transforming growth factor $\beta \beta$ (TGF- $\beta$ ), and so on. Immune function. Lamina propria lymphocytes (LPL) mainly include CD4+ T cells and SIgA+ B cells. T cells mainly modulate the immune response and can secrete down-regulation of immune responses such as IL-10 and TGF- $\beta$, and can also affect the production of SIgA by B cells. B cells exert immune function by secreting SIgA. SIgA can specifically bind to antigens, preventing antigen from invading and damaging the mucosal barrier.

\section{Immunogenicity of Intestinal Commensal Microorganisms}

There are numerous microorganisms in the intestinal tract of animals. These microorganisms live in the intestine and form a certain sense of mutual benefit and mutual restraint with the animals. These commensal microorganisms can act as antigens to stimulate specific immune cells in the intestine to activate, proliferate and differentiate, eventually producing specific antibodies or sensitizing lymphocytes to elicit an immune response. This ability to induce an immune response is called immunogenicity [5]. Symbiotic microorganisms themselves and metabolites contain a large amount of immunogenic substances. First, symbiotic microorganisms contain more nucleic acids, which are mostly non-immunogenic, but they are immunogenic after they have been combined with proteins to form nucleoproteins. Second, symbiotic microorganisms have a large number of bacterial cell walls containing peptidoglycan, teichoic acid, and lipopolysaccharide (LPS) components, some of which are immunogenic. Studies have shown that LPS, a cell wall component of Gram-negative bacteria, has a strong immunogenicity as an endotoxin, and when it acts on a large number of tissues such as the intestine, it causes an immune response. Cause inflammation and other diseases. In addition, macromolecular proteins, polysaccharides, and small-molecule polypeptides produced by the commensal microorganisms after digestion and other metabolic processes are also immunogenic. However, since the establishment process of the intestinal 
microflora is basically synchronized with that of the immune system, the animals are all sterile at the time of birth, but the microorganisms colonize the intestines within a very short time after birth, due to the immunization of newborn animals. The regulatory network of the system is relatively fragile, so microorganisms and other antigens can easily lead to life-long or long-term tolerance, which is the specific non-response reaction to the microorganisms. Under normal circumstances, due to the intestinal tolerance to symbiotic microorganisms, a large number of commensal microorganisms present in the intestine do not cause excessive immune responses. Therefore, the study and understanding of the immune tolerance mechanism of the symbiotic microorganisms in the intestine can be effectively applied to the medical control of diseases and animal nutrition, and has important application value.

\section{Intestinal Mechanisms for Immune Tolerance of Symbiotic Microorganisms}

Intestinal immune tolerance mechanisms for symbiotic microorganisms are also divided into natural immune tolerance mechanisms and acquired immune tolerance mechanisms. The intestinal tract produces natural immune tolerance to symbiotic microorganisms due to the lack of receptors recognizing certain microbial antigens or the presence of inhibitory receptors and structures on the surface of intestinal cells. At present, it has been found that Mø cells in PP knots play an important role in the formation of natural immune tolerance to symbiotic microorganisms in the intestine. Intestinal mature $\mathrm{T}$ cells and $\mathrm{B}$ cells encounter symbiotic microorganisms and other antigens to form adaptive immune tolerance. There are several types of acquired immune tolerance. First, the activation of mature $\mathrm{T}$ cells or $\mathrm{B}$ cells requires the activation of two or more signals, and when some signal factors are inhibited, T cells or B cells cannot be activated, resulting in an unresponsive state and the acquired immune tolerance. Accepted by. DC cells, Toll-like receptors (TLRs) and peroxisome proliferator-activated receptors (PPARs) $\gamma$ are involved in the formation of this type of immune tolerance. Second, when symbiotic microorganisms and other innocuous antigens are not in contact with immune cells for some reason, they are in a state of neglect, and immune tolerance can be established. The characteristics of symbiotic microorganisms and intestinal alkaline phosphatase (ALPI) play an important role in the formation of this immune tolerance. Again, activation of cell death induced by activation induced cell death (AICD) is mediated by the binding of FasL (CD178) and Fas (CD95) between T-B cells or T-T cells. T cells or B cells that are reactive to harmless antigens such as symbiotic microorganisms are eliminated. Among them, the expression levels of FasL and Fas are controlled by many factors, such as interferon and IL. Studies have suggested that the reduction of IL-2 and IL-12 levels will increase Fas-mediated antigen-specific T cell apoptosis. Finally, the role of immune regulatory cells, such as regulatory T cells (Treg cells) secrete inhibitory cytokines to form immune tolerance.

\section{Conclusion}

More and more studies are gradually elucidating the role of symbiotic microbes between humans and the intestines. It has now been clarified that intestinal microbes can affect the host's metabolism and immune pathways and participate in human health and disease. There is already evidence that not only enteric microbes have an important role in the development of gastrointestinal diseases, but also have an important influence on immune diseases (symbiotic microbes can not only provide signals that promote the development of normal immune responses, but can also affect subsequent immune responses). However, the mechanism of the interaction between intestinal symbiotic microbes and the immune system has not yet been fully understood. It may be that symbiotic microbes interact with the immune system through the production of molecular substances. If these mechanisms are clarified, the treatment of these diseases will not be blind. Instead, new antibiotics are discovered, but these bacteria are controlled by examining intestinal microbes, using prebiotics and probiotics, and they can use probiotic bacteria in the normal human body to prepare probiotics to treat some diseases. 


\section{References}

[1] ANDERSON M S, SU M A. AIRE expands: new rolesin immune tolerance and beyond [J]. Nature Reviews Immunology, 2016, 16(4):247-258.

[2] SCANDLING J D, BUSQUE S, DEJBAKHSH-JONES S, et al. Tolerance and withdrawal of immune-suppressive drugs in patients given kidney and hema-topoietic cell transplants [J]. American Journal ofTransplantation, 2012, 12(5): 1133-1145.

[3] CORREALE J, FAREZ M F. The impact of parasite infections on the course of multiple sclerosis [J]. Journal of Neuroimmunology, 2011, 233(1 / 2): 6-11.

[4] ARCK P C, HECHER K. Fetomaternal immune cross-talk and its consequences for maternal and offspring' shealth [J]. Nature Medicine, 2013, 19(5): 548-556.

[5] Wang Yongxiang, Yang Ling, Bi Jianghua, et al. Progesterone's immune tolerance in early pregnancy in ruminants [J]. Chinese Animal Husbandry and Veterinary Medicine, 2015, 42(2): 493-497 